\title{
Directed self-assembly into low-density colloidal liquid crystal phases
}

Yongxiang Gao ${ }^{1,2 *}$, Flavio Romano ${ }^{2}$, Roel P.A. Dullens ${ }^{2}$, Jonathan K. Doye ${ }^{2}$, Dirk G. A. L. Aarts ${ }^{2} *$

1. Institute for Advanced Study, Shenzhen University, Nanhai Avenue 3688, Nanshan District, Shenzhen, 518060, China

2. Department of Chemistry, Physical and Theoretical Chemistry Laboratory, University of Oxford, South Parks Road, Oxford OX1 3QZ, United Kingdom

Alignment of anisometric particles into liquid crystals (LCS) often results from an entropic competition between their rotational and translational degrees of freedom at dense packings. Here we show that by selectively functionalizing the heads of colloidal rods with magnetic nanoparticles, this tendency can be broken to direct the particles into novel, low-density LC phases. Under an external magnetic field, the magnetic heads line up in columns whereas the non-magnetic tails point out randomly in a plane perpendicular to the columns, forming bottle-brush-like objects; laterally, the bottle-brushes are entropically stabilised against coalescence. Experiments and simulations show that upon increasing the particle density the system goes from a dilute gas to a dense $2 \mathrm{D}$ liquid of bottle-brushes with a density well below the zero-field nematic phase. Our findings offer a new strategy for self-assembly into 3D open phases that may find applications in switchable photonics, filtration and light-weight materials.

Liquid crystals are states of matter formed by highly anisometric molecules, which display long-range orientational alignment yet no or only partial positional periodicity [1]. Their physical attributes lie between those of a liquid and those of a crystalline solid, and this duality gives rise to peculiar material properties, which has spurred a myriad of high-tech applications besides display devices [2]. The development of LC-forming materials in the colloidal rather than the molecular domain has been an ongoing effort that involves a range of model systems [3], including elongated biological virus particles [4], $\beta$-ferric oxyhydroxide [5], boehmite [6] and silica rods [7]. The underlying incentive is multi-fold: on one hand they serve as model systems to understand the LC phase transition, dynamics and rheology; on the other hand, they emerge as new colloidal materials with potential new applications [8]. Most of these systems can be modelled as hard rods, whose 
disorder-to-order transition is entropy-driven where the apparent loss of orientational entropy is compensated by the gain in translational free volume $[9,10]$. As a result, the structure of the system is dictated by the particle shape, which favours efficient dense packings. Assembly into low-density liquid crystal phases is therefore challenging, despite the potential applications of such innovative 3D architectures in many technological sectors [11-13].

Self-assembly of Janus (or patchy) colloids, that is colloids with patterned sticky sites [14], provides a conceptually conceivable solution to this challenge. For example, computer simulations have predicted that patchy colloids can reach targeted intricate, nonconventional structures that are predefined in the particle design [15]. This is achieved by the dominant role played by the potential energy between the sticky sites in minimizing the free energy. Translating this concept into reality by intelligently designing and crafting colloids through site-specific chemistry represents a central challenge in materials science [16]. Most of the current experimental efforts have been focused on synthesizing such chemically structured colloids. Yet, there are very limited examples where extended superstructures can be self-assembled, and most are constrained to 2D [17]. 3D analogues, which pose additional challenges in upscaling of the synthesis $[18,19]$, stability against gravitational stress [20], and optical imaging, are yet to be realized.

Here we expand the design space of self-assembling Janus particles to encompass low-density colloidal liquid crystals. Specifically, we design our colloidal building blocks as rods with one end magnetic, such that the site-specific magnetic interactions will dominate the self-assembly. We choose magnetic interactions due to the ease of tuning their strength and the external field direction (e.g. parallel or perpendicular to the gravitational field), and their insensitivity to the solvent. The latter freedom allows a solvent to be selected whose refractive index is close to that of the particles to facilitate imaging in 3D by confocal microscopy.

Experimentally, we produce silica rods with one end selectively doped with magnetite $\left(\mathrm{Fe}_{3} \mathrm{O}_{4}\right)$ nanoparticles. We realize this particle design by emulsifying an aqueous dispersion of polyvinylpyrolindone (PVP)-functionalized $\mathrm{Fe}_{3} \mathrm{O}_{4}$ nanoparticles into pentanol, and subsequently 
triggering the hydrolysis and condensation reactions of tetraethyl orthosilicate (TEOS) for directional growth of silica. The $\mathrm{Fe}_{3} \mathrm{O}_{4}$ nanoparticles are locked within the precipitated silica matrix at the beginning, and are essentially absent in the latter growth of the silica rods. Meanwhile, the rods are labeled with a dye for fluorescence imaging. The synthesis protocol is sketched in Fig. 1(a), inspired

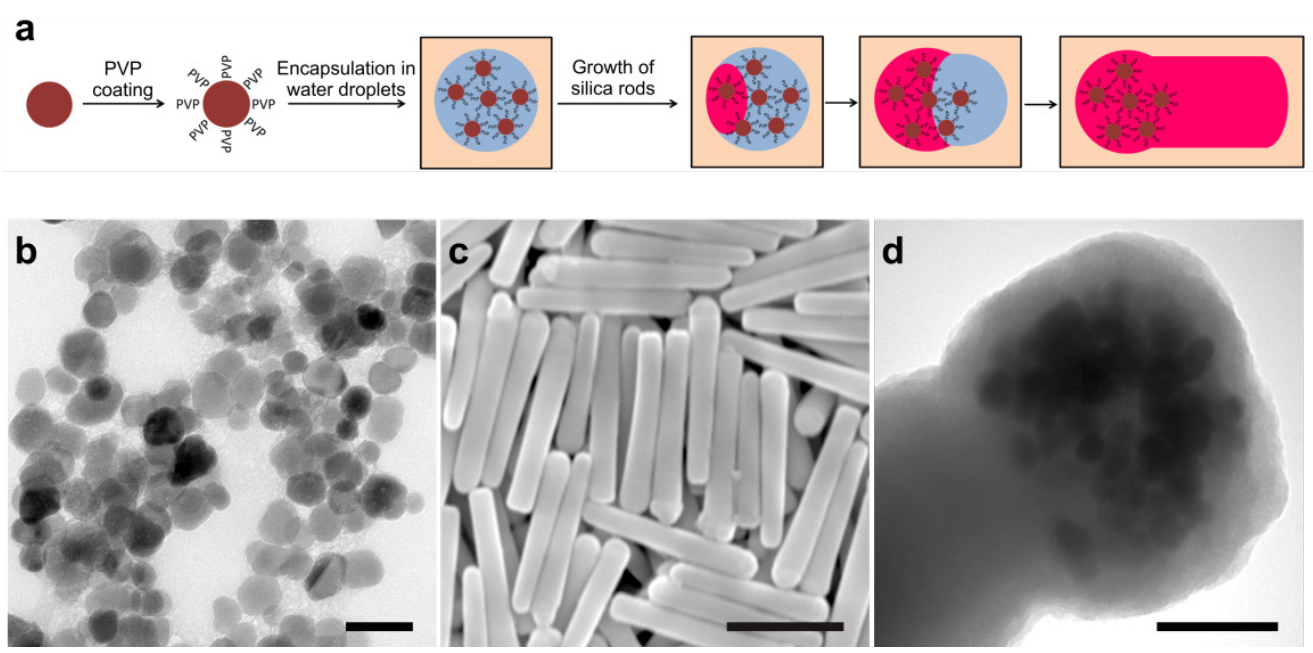

FIG. 1. Fabrication of colloidal magnetic Janus rods. (a) Schematic showing the synthesis steps, which include surface functionalization of $\mathrm{Fe}_{3} \mathrm{O}_{4}$ nanoparticles by PVP absorption, encapsulation of the resulted nanoparticles in water droplets, and directional growth of silica by successive hydrolysis and condensation reactions of TEOS. (b) TEM of $\mathrm{Fe}_{3} \mathrm{O}_{4}$ nanoplarticles. (c) SEM of the synthesized rods. (d) TEM shows that $\mathrm{Fe}_{3} \mathrm{O}_{4}$ nanoparticles are located in the head of a rod. Scalebars are $50 \mathrm{~nm}, 2 \mu \mathrm{m}$ and $100 \mathrm{~nm}$ in (b)-(d).

the synthesis scheme of Kuijk et al [7]. The diameter of the $\mathrm{Fe}_{3} \mathrm{O}_{4}$ nanoparticles is $26 \pm 6 \mathrm{~nm}$ (Fig. 1b), revealed by transmission electron microscopy (TEM). An additional silica layer was added to the particles through seeded growth to increase the particle diameter and thus facilitate imaging at single-particle resolution. Scanning electron microscopy (SEM) shows that the synthesized particles are slightly tapered (Fig. 1c), with dimensions of $3.5 \pm 0.4$ (Length) $\times 0.44 \pm 0.03$ (mid-Width) $\times$ $0.50 \pm 0.04$ (Head) $\mu \mathrm{m}^{3}$, measured from approximately 100 particles. The head of each rod was successfully doped with $\mathrm{Fe}_{3} \mathrm{O}_{4}$ nanoparticles, as confirmed by TEM (Fig. 1d) and bright-field optical microscopy (Fig. S1) [21]. These particles have been shown to possess a weak remnant magnetic 
moment pointing perpendicular to the rod's orientation [22]. Note that these particles are charge stabilized and the dipolar interaction between the remnant magnetic moments of particles are too weak to induce binding without an external magnetic field. To facilitate 3D confocal microscopy, we suspend the rods in dimethylformamide (DMF), a solvent with a refractive index close to that of silica. Further details of the system are given in the Methods [21].

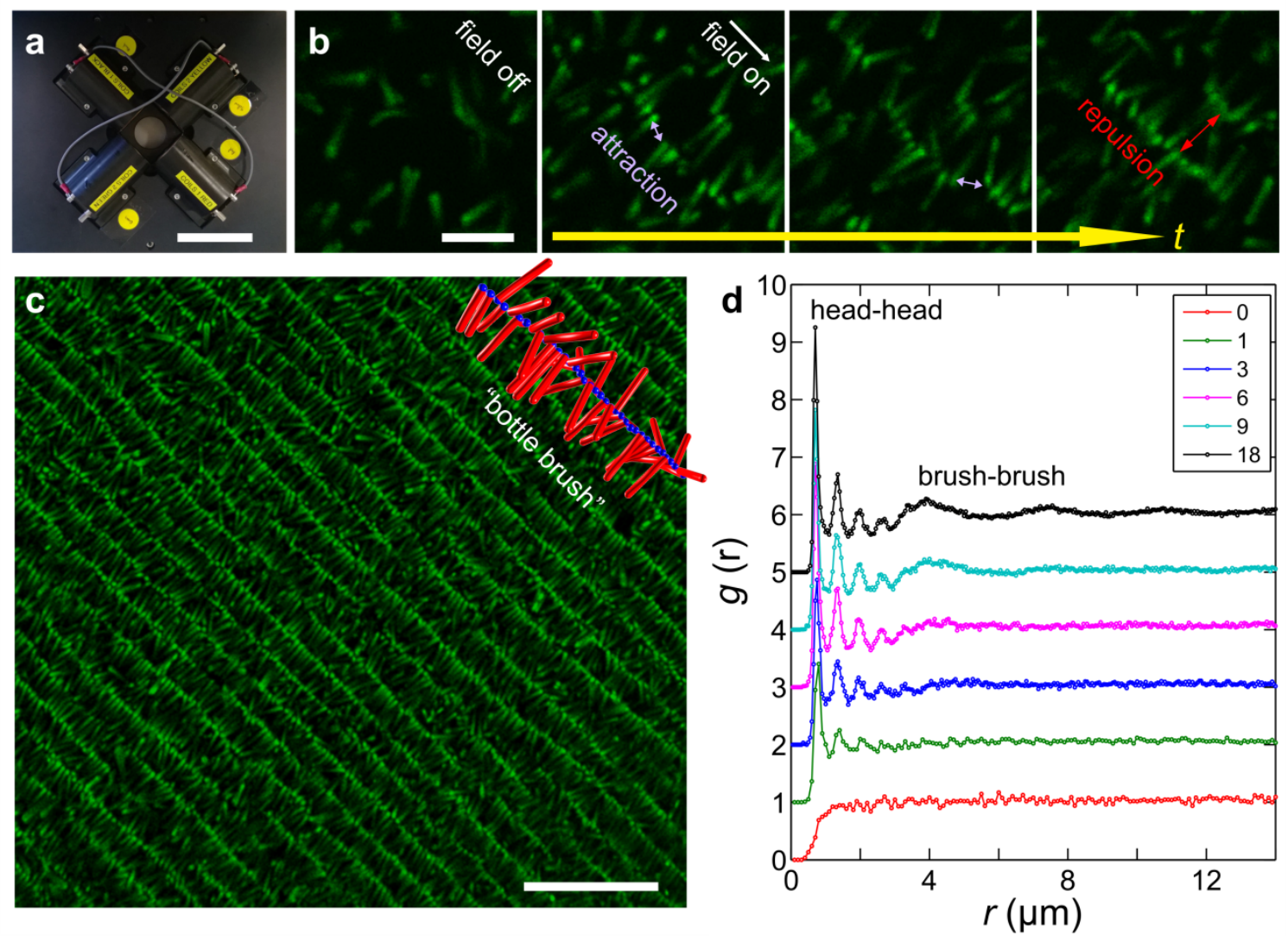

FIG. 2. Bottle brush formation. (a) Magnetic field setup mounted on a home-built microscope stage for applying an in-plane magnetic field. (b) A time series of confocal images showing the self-assembly pathway, directed by an external magnetic field. (c) A quasi-2D periodic stacking of the self-assembled bottle-brushes. Inset is a computer-rendered bottle-brush. (d) The evolution of the RDFs of the magnetic heads as a function of time (in units of minutes). The plots are shifted vertically for clarity. Scalebars are $10 \mathrm{~cm}, 5 \mu \mathrm{m}$ and $20 \mu \mathrm{m}$ in (a)-(c).

To examine the self-assembly pathway of these particles, we start our experiment from the isotropic phase and switch on a magnetic field (150 Gauss) parallel to the imaging plane 
(perpendicular to gravity) using the setup shown in Fig. 2(a). The fluorescence of the particles gradually decreases from head to tail [7] (Fig. 2b,c), offering a natural way to identify their polarity. At zero magnetic field, the rods display no positional or orientational order, see Fig.2b (field off). When the external field is switched on, the particles first orient themselves perpendicular to the field; subsequently, the site-specific dipolar attraction, resulting from the sum of the remnant and the induced moments, becomes strong enough to line up the heads and form chains along the field direction, see Supplementary Movie 1 [21] and Fig. 2b (field on). The non-magnetic tails point out randomly in a plane perpendicular to the chain direction and undergo angular thermal fluctuations. We name these structures colloidal "bottle brushes" due to their resemblance to the molecular analogue [23]. The tendency of the tails to randomize orientation provides an effective repulsive entropic barrier, stabilizing the bottle brushes from lateral aggregation (see Fig. 2b). Eventually the system forms a quasi-2D periodic stacking of the brushes that extends over several hundred micrometers (a subset is shown in Fig. 2 c for clarity). To quantitatively capture this process, we have localized the positions of the magnetic heads with a standard centroid finding algorithm [24] (see Fig. S2a) [21] and computed the radial distribution function (RDF), $g(r)$. At zero field, the RDF displays no structure features, Fig. 2(d) ( $t=0)$. With the applied field, the RDF first develops peaks that are due to the head-head correlation, see Fig. 2(d) ( $t=1 \mathrm{~min})$. The first peak is located at $r=0.7 \mu \mathrm{m}$, slightly larger than the diameter of the magnetic head, which we attribute to the surface charge of the particles. The brushes themselves behave as soft cylinders due to their open low-density nature. As the system concentrates, the brushes become partially interpenetrated and the system gradually develops structural correlations at a larger length scale that is comparable to the bottle brush size. This is evident from Fig. 2(d) ( $t=3,6,9$ and 18 mins) and at long time ( $t=18$ mins) multiple peaks developed in the RDFs, which reflect a growing correlation length scale associated with the bottle-brushes. Note that the intra-brush distance $(\sim 4 \mu \mathrm{m})$ is much less than the diameter $(\sim 6.5 \mu \mathrm{m})$ of a single brush.

To further investigate the self-assembled structures in 3D, we apply a magnetic field parallel to 

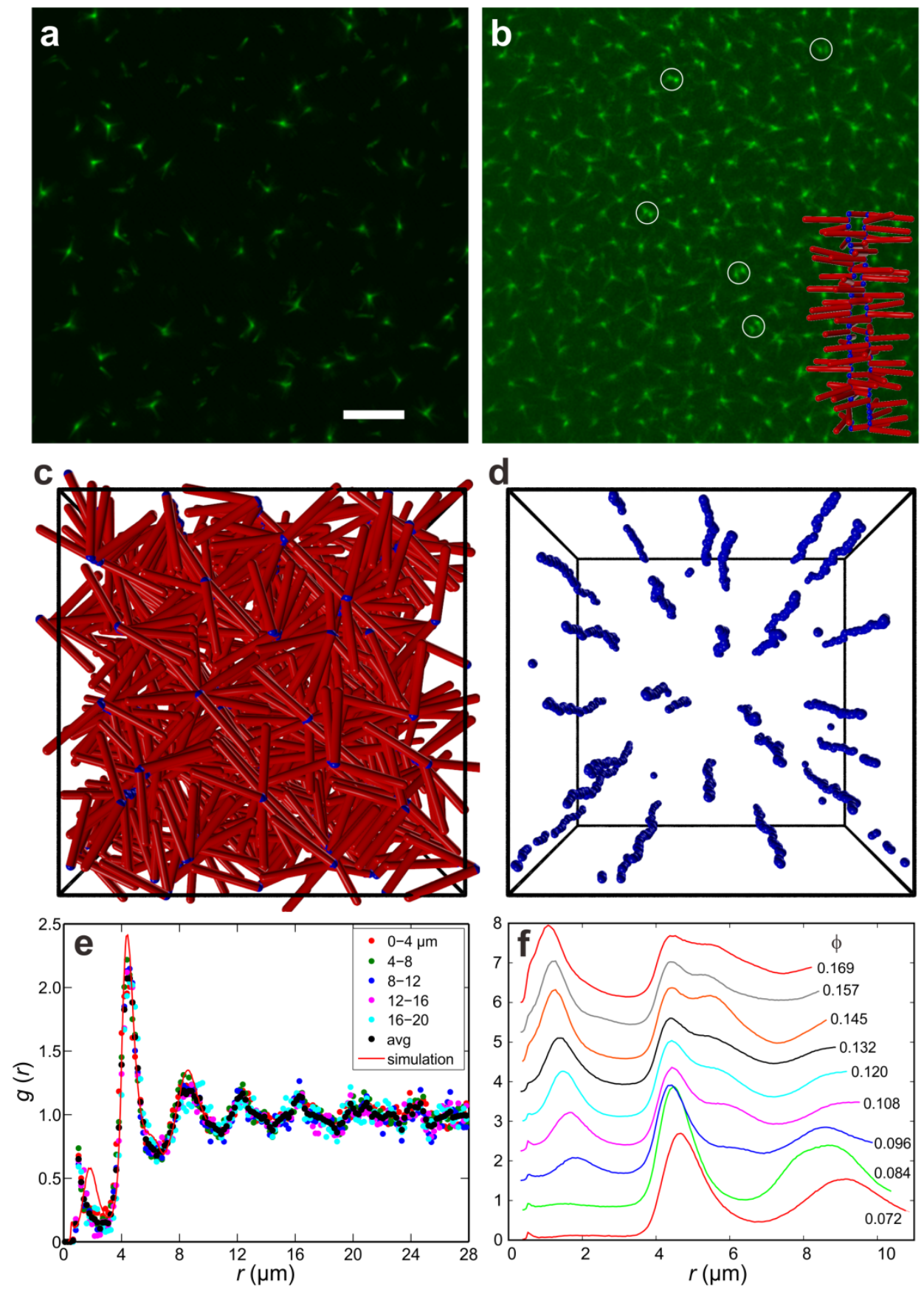

FIG. 3. 3D self-assembly from the isotropic phase. (a)-(b), Top views of bottle brushes at $\phi^{\sim} 0.007$ and 0.06, respectively. The scalebar is $10 \mu \mathrm{m}$. In (b), a few double brushes are highlighted by circles. The inset shows a rendered configuration of a double brush extracted from simulations. (c)-(d), Snapshots of the simulated bottle brush phase (with (d) only showing the magnetic heads) at a similar condition to (b). (e) RDFs of bottle brushes at $\phi \sim 0.06$ together with simulation results. (f) RDFs of simulated bottle brushes at a field strength of 150 Gauss. 
gravity (perpendicular to the imaging plane) on the particles (Fig. 3 and Supplementary Movie 2) [21]. The cross sections of the formed structures at two different packing volume fractions are highlighted in Fig. 3(a)-(b). This again illustrates that rods are oriented perpendicular to the backbone of the bottle brushes in a random direction. At $\phi^{\sim} 0.007$, the bottle-brushes are in a gas-like phase, where the chains are arranged randomly. At a higher volume fraction, $\phi \sim 0.06$, the system forms a dense fluid of bottle brushes. The dipolar interaction between strings of magnetic particles is very short ranged [25]; thus the bottle-brushes effectively interact entropically, as outlined earlier. Rendering of the 3D confocal data (Supplementary Movie 3) [21] clearly reveals the packing of these bottle-brushes.

To quantitatively characterize the structure of the dense phase, we perform image analysis [24] to localize the centroid or the backbone position of each bottle-brush (Fig. S2) [21]. The RDF calculated from the brush positions reveals two important features (Fig. 3e). One is the oscillation of peaks at large distances ( $\sim \mu \mathrm{m}$ and beyond), which is a typical feature of a dense liquid, signaling the brush-brush correlation. The other is the emergence of a peak at a distance that is much smaller than the pair distance between brushes, which is due to doubly occupied bottle-brushes (Fig. 3b). To further examine the structural order, we perform Voronoi tessellation (Fig. S3) [21], considering each double bottle brush as one brush. We observe isolated five-fold and seven-fold defects, which again is a typical feature of a 2D dense fluid. Since each rod orients approximately perpendicular to the alignment (field) direction, the orientational order parameter quantifying nematic order, defined as $S=\frac{1}{2}\left\langle 3 \cos ^{2} \theta-1\right\rangle$, will tend to $-\frac{1}{2}$. This is a clear example of anti-nematic behavior [26], here illustrated for the first time for a system of colloidal rods. We highlight the fact that the volume fraction of this low-density liquid crystalline phase is well below the volume fraction of 0.3 , at which hard rods with a similar aspect ratio show nematic order [10].

To provide further microscopic insight and to explore the state space of this system more fully, we performed Monte Carlo computer simulations of these particles (see Supplementary Materials for the details of the model used) [21]. The simulations further revealed the robust tendency of the 
system to form bottle-brushes over a wide range of densities (Fig. 3f), as exemplified by the configuration in Fig. 3(c)-(d), and showed quantitative agreement with the details of the experimental structure (e.g. the inter-brush spacing in Fig.3e). The simulations also allow the value of $S$ to be computed directly, which is -0.49 , confirming the strong anti-nematic ordering.

One interesting feature is that once the density is sufficient for the brushes to contact, the inter-brush spacing is relatively insensitive to further increasing the density. Instead, the density increase is accommodated by the coalescence of the brushes. A resulting "double bottle brush" is illustrated in Fig. 3(b) inset with the rods associated with each chain tending to be restricted to separate half-cylinders. A number of such double chains can be seen in the experimental and simulation configurations in Fig. $3(\mathrm{~b})-(\mathrm{c})$, giving rise to the small peak in the radial distribution function at $1 \mu \mathrm{m}$ (Fig. 3e). A very similar type of clustering has been predicted for systems interacting with suitably designed soft repulsions [27] and has received much attention [28], but as yet there has been no experimental soft matter realization of such a phenomenon. The observed condensation of chains is consistent with the soft character of the entropic repulsion between the bottle brushes, albeit with a hard repulsion at very short range as the chains of magnetic heads cannot overlap.

To interrogate what new phases may emerge in the high density regime, we first let the rods sediment into a smectic phase before applying a magnetic field (Fig. 4a). A field of 175 Gauss is then switched on and off for about two hours to help the system relax before eventually leaving the field on. We discover that the magnetic heads stack into a single plane with the tails randomly sticking out to each side of the plane (Fig. 4b-c and Supplementary Movie 4) [21], effectively forming the equivalent of a 2D planar or layer bottle-brush. The layers further interpenetrate and stack into one-dimensional periodically stacked superstructures, forming an unusual smectic lamellar phase. The 3D nature of the new liquid crystalline phase is clearly revealed in the orthogonal views of a smaller region of the 3D confocal image (Fig. 4c). A larger field of view of the assembled structure is shown in Fig. S4 [21]. Here, we stress again the importance of entropic and steric effect from the tails in stabilizing the structure; by contrast, the magnetic particles on their own would be expected to 
form a percolated network or a traditional close packed crystal [29] at high density.
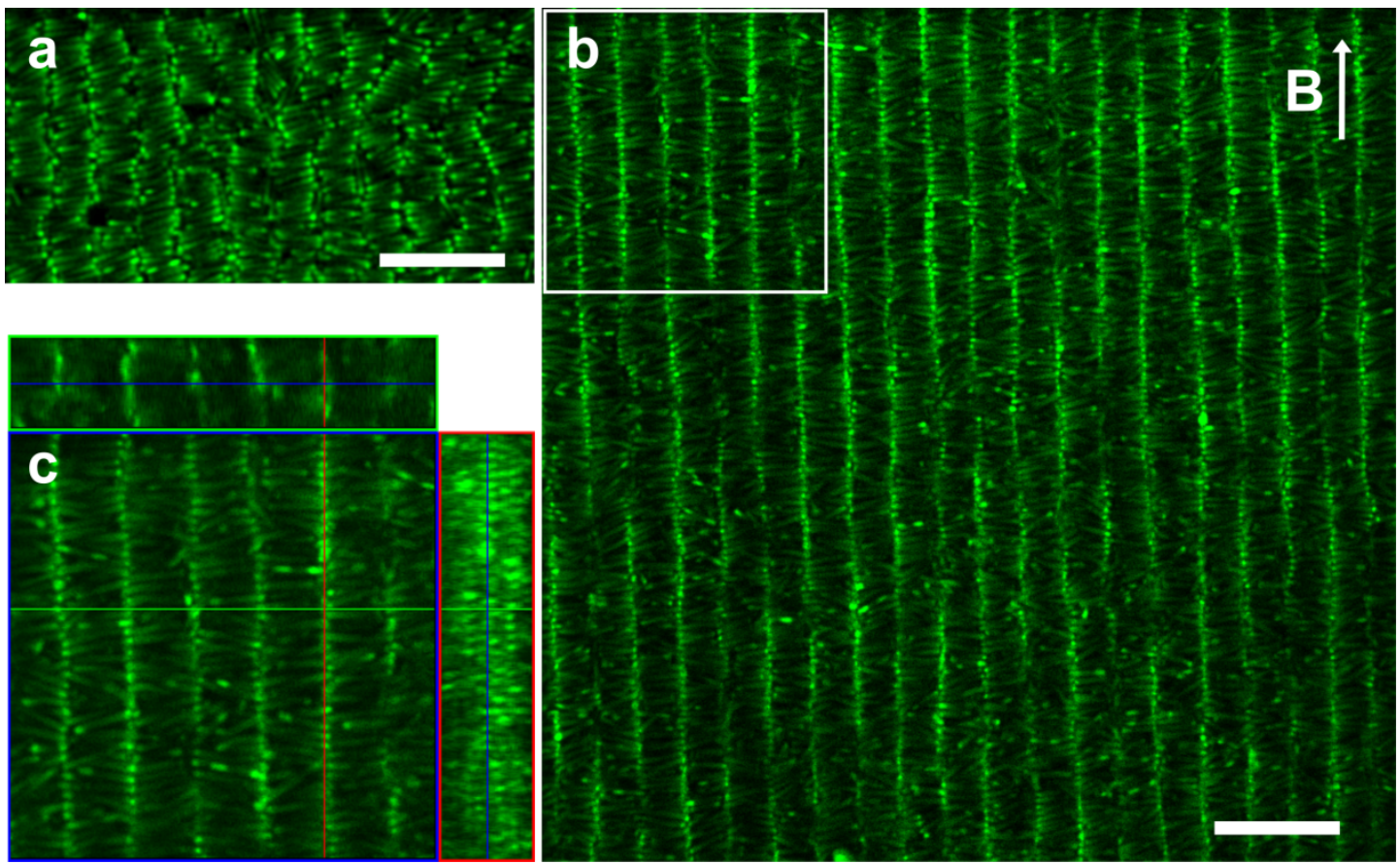

FIG. 4. 3D self-assembly from a dense smectic phase. (a) The system forms a smectic phase at the bottom of the sample at $B=0$. (b) The system self-assembles into a new liquid crystal of periodically stacked layers at B=175 Gauss. (c) A zoomed-in view of a small area highlighted in (b) with orthogonal views along the highlighted lines to further illustrate the structure. Scalebars represent $10 \mu \mathrm{m}$.

In summary, we have designed and realized a colloidal model system consisting of rods with a magnetic head that shows tunable 3D bulk self-assembly that can be followed at the single particle level. The interplay between dipolar interactions, which favour the lining up of the heads along the field direction, and entropy, which causes the tails to point out randomly in a plane perpendicular to the columns, leads to an interesting self-assembly pathway. This unique feature leads to a hierarchical self-assembly, which organizes colloids into a 3D low-density liquid crystalline phase of densely packed bottle brushes with anti-nematic characteristics. This occurs at a density well below where nematic order starts to emerge in hard rods. Furthermore, we reveal at high density a novel smectic lamellar phase composed of periodically stacked layers. These novel 3D superstructures arise from tailoring the colloidal architecture with both an anisotropic geometrical shape and a site-specific directional attraction $[18,30]$. Our results point to a new strategy for self-assembly into 
open or even empty structures, through a combination of the large excluded volume effect from particles with high aspect ratios and hierarchical build-up mechanisms.

We acknowledge David Dunmur for stimulating discussions. Y. G. acknowledges financial support from Marie Curie actions (FP7-PEOPLE-2012-IIF No 327919) and Shenzhen University.

Correspondence and requests for materials should be addressed to Y.G. and D.G.A.L..

*e-mail: yongxiang.gao@szu.edu.cn; dirk.aarts@chem.ox.ac.uk

\section{References}

[1] M. J. Stephen, and J. P. Straley, Rev. Mod. Phys. 46, 617 (1974).

[2] J. W. G. Goodby, P. J. Collings, T. Kato, C. Tschierske, H. F. Gleeson, and E. P. Raynes, Handbook of liquid crystals (2014), Second completely revised and greatly enlarged edition. edn., Vol. 8. Applications of Liquid Crystals, Applications of Liquid Crystals, 8.

[3] H. N. Lekkerkerker, and G. J. Vroege, Philos. Trans. A Math. Phys. Eng. Sci. 371, 20120263 (2013).

[4] Z. Dogic, and S. Fraden, Curr. Opin. Colloid \& Interface Sci. 11, 47 (2006).

[5] Y. Maeda, and S. Hachisu, Colloids and Surfaces 6 (1983).

[6] P. A. Buining, and H. N. W. Lekkerkerker, J. Phys. Chem. 97, 11510 (1993).

[7] A. Kuijk, A. van Blaaderen, and A. Imhof, J. Am. Chem. Soc. 133, 2346 (2011).

[8] M. Fu, K. Chaudhary, J. G. Lange, H. S. Kim, J. J. Juarez, J. A. Lewis, and P. V. Braun, Adv. Mater. 26, 1740 (2014).

[9] L. Onsager, Ann. N. Y. Acad. Sci. 51 (1949).

[10] P. Bolhuis, and D. Frenkel, J. Chem. Phys. 106, 666 (1997).

[11] J. F. Galisteo-Lopez, M. Ibisate, R. Sapienza, L. S. Froufe-perez, A. Blanco, C. Lopez, Adv. Mater. 23, 30 (2011).

[12] O. D. Velev, and E. W. Kaler, Adv. Mater. 12, 531 (2000).

[13] J. de Vicente, D. J. Klingenberg, and R. Hidalgo-Alvarez, Soft Matter 7, 3701 (2011).

[14] S. C. Glotzer, Science 306, 419 (2004).

[15] Z. L. Zhang, and S. C. Glotzer, Nano letters 4, 1407 (2004).

[16] S. C. Glotzer, and M. J. Solomon, Nat. Mater. 6, 557 (2007).

[17] Q. Chen, S. C. Bae, and S. Granick, Nature 469, 381 (2011).

[18] F. Li, D. P. Josephson, and A. Stein, Angew. Chem. 50, 360 (2011).

[19] A. B. Pawar, and I. Kretzschmar, Macromol. Rapid Commun. 31, 150 (2010).

[20] J. Zhang, E. Luijten, and S. Granick, Annu. Rev. Phys. Chem. 66, 581 (2015).

[21] See Supplemental Material for Methods, Characterization, and Movies.

[22] Y. Gao, A. K. Balin, R. P. A. Dullens, J. M. Yeomans, and D. G. A. L. Aarts, Phys. Rev. Lett. 115, 248301 (2015)

[23] R. Verduzco, X. Li, S. L. Pesek, and G. E. Stein, Chem. Soc. Rev. 44, 2405 (2015).

[24] Y. Gao, and M. L. Kilfoil, Opt. Express 17, 4685 (2009).

[25] E. M. Furst, and A. P. Gast, Phys. Rev. E 62, 6916 (2000).

[26] I. Dozov, E. Paineau, P. Davidson, K. Antonova, C. Baravian, I. Bihannic, L. J. Michot, J. Phys. Chem. B 115, 7751 (2011).

[27] B. M. Mladek, D. Gottwald, G. Kahl, M. Neumann, C. N. Likos, Phys. Rev. Lett. 96, 045701 (2006).

[28] F. Sciortino, and E. Zaccarelli, Nature 493, 30 (2013).

[29] J. W. Swan, J. L. Bauer, Y. Liu, and E. M. Furst, Soft Matter 10, 1102 (2014).

[30] G. R. Yi, D. J. Pine, and S. Sacanna, J. Phys. Condens. Matter 25, 193101 (2013).

[31] B. Zhao, H. Zhou, C. Liu, Y. Long, G. Yang, C. Tung, K. Song, New J. Chem. 40, 6541 (2016). 